\title{
THE PRINCIPLES \\ OF INTERNATIONAL HUMANITARIAN LAW ${ }^{1}$
}

\author{
by \\ Jean Pictet \\ Director-General, International \\ Committee of the Red Cross \\ Lecturer at Geneva University
}

In this sixty-page book the writer defines fully and with concision humanitarian law in its widest sense, the laws of war of The Hague and Geneva, and the principles which form the basis for this humanitarian law.

This clear summary is understandable to everybody interested in humanitarian ideas and actions in the world today. In addition, the appendix is a chart of the principles of humanitarian law.

It will be recalled that an earlier work by this author, The Principles of the Red Cross, gives the general reader a clear exposition of its subject. Copies of this book, which has already had considerable success, are available in French, English, German and Spanish, from the ICRC Geneva, which published the book.

1 The Principles of International Humanitarian Law can be obtained from the ICRC, 7 avenue de la Paix, 1211 Geneva (postal cheque account No. 12-5527). Cost Sw.fr. 8.- . 


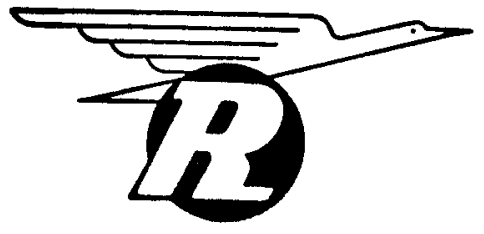

\section{H. Rifschard\&Gie.S.A.}

\section{INTERNATIONAL TRANSPORT TRAVEL AGENCY}

GENEVA, 49, route des Jeunes

Telephone 437600 - Teleprinter 22167

Travel agency: Branch office in Geneva:

18, place Cornavin - $\varnothing 323402$

\section{Exchange - Tickets - Sea passages \\ Insurance - Customs Agency \\ Road haulage - Storage}

Home delivery of air and rail tickets on request by telephone

Branches :

LAUSANNE - ANNEMASSE (France) 


\section{RHÔNE HOTELS INTERNATIONAL S.A. GENEVA}

anticipate the modern businessman's desires:

GENEVA

HOTEL DU RHÔNE

First class

Tel. 319831 Cables Rhonotel

Teleprint 22213

ANKARA GRAND HOTEL ANKARA First class

(Büyük Ankara Oteli)

Tel. 171106 Cables Grantel Teleprint 98

and his family's vacation plans:

\section{PORTUGAL HOTEL ALGARVE De Luxe}

Open all year round

Praia da Rocha (Portimâo)

Tel. 1101/12 Cables Algarvotel

SWISS MANAGEMENT: c/O HOTEL DU RHÔNE - GENEVA
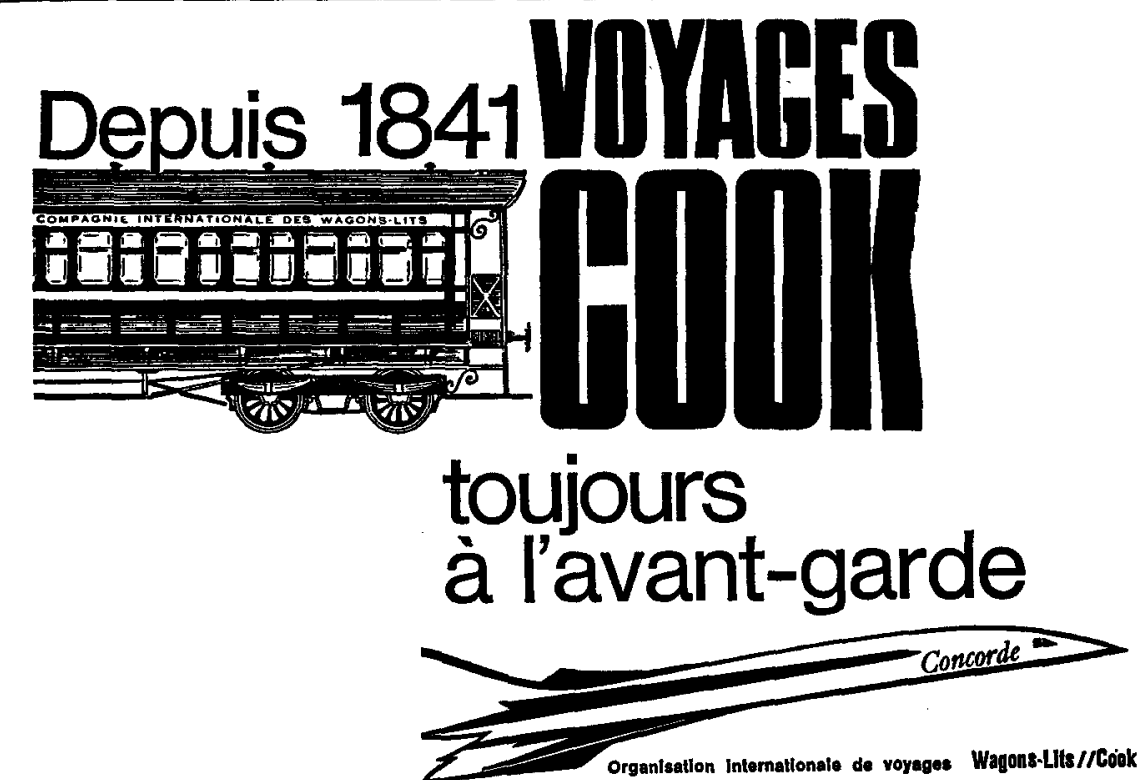

Tú 312130

1002 Lausanne-Ville

1001 Lausanne-Gare

Grand-Chene 7

Tol. 2272

1820 Montreux

Avenue du Casino 4

T61. 227218

Tel. 612860 
AFGHANISTAN - Afghan Red Crescent, Kabul.

ALBANIA - Albanian Red Cross, 35, Rruga Barrikadavet, Tirana.

ALGERIA - Central Committee of the Algerian Red Crescent Society, 15 bis Boulevard Mohamed V, Algiers.

ARGENTINE - Argentine Red Cross, H. Yrigoyen 2068, Buenos Aires.

AUSTRALIA - Australian Red Cross, 122-128 Flinders Street, Melbourne, C. $t$.

AUSTRIA - Austrian Red Cross, 3 Gusshausstrasse, Postfach 39, Vienna IV.

BELGIUM - Belgian Red Cross, 98, Chaussée de Vleurgat, Brussels 5.

BOLIVIA - Bolivian Red Cross, Avenida Simon Bolivar, 1515 (Casilla 741), La Pas.

BRAZIL - Brazilian Red Cross, Praça da Cruz Vermelha 10-12, Caixa postal $1286 \mathrm{ZC} / 00$, Rio de Janeiro.

BULGARIA - Bulgarian Red Cross, 1, Boul. S.S. Biruzov, Sofia.

BURMA - Burma Red Cross, 42, Strand Road, Red Cross Building, Rangoon.

BURUNDI - Red Cross Society of Burundi, rue du Marché 3, P.O. Box 1324, Bujumbura.

CAMBODIA - Cambodian Red Cross, 17, Vithei Croix-Rouge, P.O.B. 94, Phnom-Penh.

CAMEROON - Central Committee of the Cameroon Red Cross Society, rue HenryDunant, P.O.B. 631, Yaoundé.

CANADA - Canadian Red Cross, 95 Wellesley Street, East, Toronto 284, (Ontario).

CEYLON - Ceylon Red Cross, 106 Dharmapala Mawatte, Colombo VII.

CHILE - Chilean Red Cross, Avenida Santa Maria 0150, Casilla 246 V., Santiago de Chile.

CHINA - Red Cross Society of China, 22 Kanmien Hutung, Peking, $E$.

COLOMBIA - Colombian Red Cross, Carrera 7a, 34-65 Apartado nacional 1110, Bogota D.E.

CONGO - Red Cross of the Congo, 41, Avenue Valcke, P.O. Box 1712, Kinshasa.

COSTA RICA - Costa Rican Red Cross, Calle 5a Apartado 1025, San José.

CUBA - Cuban Red Cross, Ignacio Agramonte, 461, Havana.

CZECHOSLOVAKIA - Czechoslovak Red Cross, Thunovska 18, Prague I.

DAHOMEY - Red Cross Society of Dahomey, P.O. Box 1, Porto Novo.

DENMARK - Danish Red Cross, Ny Vestergade 17, Copenhagen $K$.

DOMINICAN REPUBLIC - Dominican Red Cross, Calle Galvan 24, Apartado 1293, Santo Domingo.

ECUADOR - Ecuadorean Red Cross, Calle de la Cruz Roja y Avenida Colombia 118, Quito.

ETHIOPIA - Ethiopian Red Cross, Red Cross Road No. 1, P.O. Box 195, Addis Ababa.

FINLAND - Finnish Red Cross, Tehtaankatu $1 \mathrm{~A}$, Box 14168, Helsinki 14.
FRANCE - French Red Cross, 17, rue QuentinBauchart, Paris ( $\left.{ }^{\circ}\right)$.

GERMANY (Dem. Republic) - German Red Cross in the German Democratic Republic, Kaitzerstrasse 2, Dresden A. 1.

GERMANY (Federal Republic) - German Red Cross in the Federal Republic of Germany, Friedrich-Ebert-Allee 71, 5300 Bonn 1, Postfach (D.B.R.).

GHANA - Ghana Red Cross, P.O. Box 835, Accra.

GREAT BRITAIN - British Red Cross, 9 Grosvenor Crescent, London, S.W.1.

GREECE - Hellenic Red Cross, rue Lycavittou 1, Athens 135.

GUATEMALA - Guatemalan Red Cross, 3.0 Calle 8-40 zona 1, Guatemala C.A.

GUYANA - Guyana Red Cross, P.O. Box 351, Eve Leary, Georgetown.

HAITI - Haiti Red Cross, Place des Nations Unies, B.P. 1337, Port-au-Prince.

HONDURAS - Honduran Red Cross, Calle Henry Dunant 516, Tegucigalpa.

HUNGARY - Hungarian Red Cross, Arany Janos utca 31, Budapest $V$.

ICELAND - Icelandic Red Cross, Ølduggøtu 4, Reykjavik, Post Box 872.

INDIA - Indian Red Cross, 1 Red Cross Road, New Delhi 1.

INDONESIA - Indonesian Red Cross, Tanah Abang Barat 66, P.O. Box 2009, Djakarta.

IRAN - Iranian Red Lion and Sun Society, Avenue Ark, Teheran.

IRAQ - Iraqi Red Crescent, Al-Mansour, Baghdad.

IRELAND - Irish Red Cross, 16 Merrion Square, Dublin 2.

ITALY - Italian Red Cross, 12, via Toscana, Rome.

IVORY COAST - Ivory Coast Red Cross Society, B.P. 1244, Abidjan.

JAMAICA - Jamaica Red Cross Society, 76 Arnold Road, Kingston 5.

JAPAN - Japanese Red Cross, 5 Shiba Park, Minato-Ku, Tokyo.

JORDAN - Jordan National Red Crescent Society, P.O. Box 10001, Amman.

KENYA - Kenya Red Cross Society, St Johns Gate, P.O. Box 712, Nairobi.

KOREA (Democratic People's Republic) - Red Cross Society of the Democratic People's Republic of Korea, Pyongyang.

KOREA (Republic) - The Republic of Korea National Red Cross, 32-3 Ka Nam San-Donk, Seoul.

KUWAIT - Kuwait Red Crescent Society, P.O. Box 1359, Kuwait.

LAOS - Lao Red Cross, P.B. 650, Vientiane.

LEBANON - Lebanese Red Cross, tue Général Spears, Beirut.

LIBERIA - Liberian National Red Cross, National Headquarters, Corner of Tubman boulevard and 9 th Street Sinkor, P.O. Box 226, Monrovia. 


\section{ADDRESSES OF CENTRAL COMMITTEES}

LIBYA - Libyan Red Crescent, Berka Omar Mukhtar Street, P.O. Box 541, Benghaxi.

LIECHTENSTEIN - Liechtenstein Red Cross, Vaduz.

LUXEMBURG - Luxemburg Red Cross, Parc de la Ville, C.P. 234, Luxemburg.

MADAGASCAR - Red Cross Society of Madagascar, rue Clemenceau, P.O. Box 1168, Tananarive.

MALAYSIA - Malaysian Red Cross Society, 519 Jalan Belfield, Kuala Lumpur.

MALI - Mali Red Cross, B.P. 280, route de Koulikora, Bamako.

MEXICO - Mexican Red Cross, Avenida Ejército Nacional, no 1032, Mexico 10, D.F.

MONACO - Red Cross of Monaco, 27 Boul. de Suisse, Monte-Carlo.

MONGOLIA - Red Cross Society of the Mongolian People's Republic, Central Post Office, Post Box 537, Ulan Bator.

MOROCCO - Moroccan Red Crescent, rue Benzakour, B.P. 189, Rabat.

NEPAL - Nepal Red Cross Society, Tripureswore, P.B. 217, Kathmandu.

NETHERLANDS - Netherlands Red Cross, 27 Prinsessegracht, The Hague.

NEW ZEALAND - New Zealand Red Cross, 61 Dixon Street, P.O.B. 6073, Wellington C.2.

NICARAGUA - Nicaraguan Red Cross, 12 Avenida Noroeste, Managua, D.N.

NIGER - Red Cross Society of Niger, B.P. 386, Niamey.

NIGERIA - Nigerian Red Cross Society, Eko Akete Close, off. St. Gregory Rd., Onikan, P.O. Box 764, Lagos.

NORWAY - Norwegian Red Cross, Parkveien 33b, Oslo.

PAKISTAN - Pakistan Red Cross, Frere Street, Karachi 4.

PANAMA - Panamanian Red Cross, Apartado 668, Panama.

PARAGUAY - Paraguayan Red Cross, calle André Barbero y Artigas 33, Asuncion.

PERU - Peruvian Red Cross, Jiron Chancay 881, Lima.

PHILIPPINES - Philippine National Red Cross, 860 United Nations Avenue, P.O.B. 280, Manila.

POLAND - Polish Red Cross, Mokotowska 14, Warsaw.

PORTUGAL - Portuguese Red Cross, General Secretaryship, Jardim 9 de Abril, 1 a 5, Lisbon 3.

RUMANIA - Red Cross of the Socialist Republic of Rumania, Strada Biserica Amzei 29, Bucarest.

SALVADOR - Salvador Red Cross, 3a Avenida Norte y 3a Calle Poniente 21, San Salvador.

SAN MARINO - San Marino Red Cross, Palais gouvernemental, San Marino.
SAUDI ARABIA - Saudi Arabiau Red Crescent, Riyadh.

SENEGAL - Senegalese Red Cross Society, Bld. Franklin-Roosevelt, P.O.B. 299, Dakar.

SIERRA LEONE - Sierra Leone Red Cross Society, 6 Liverpool Street, P.O.B. 427, Freetown.

SOMALI REPUBLIC - P.O. Box 937, Mogadiscio.

SOUTH AFRICA - South African Red Cross, Cor. Kruis \& Market Streets, P.O.B. 8726, Johannesburg.

SPAIN - Spanish Red Cross, Eduardo Dato 16, Madrid, 10.

SUDAN - Sudanese Red Crescent, P.O. Box 235, Khartoum.

SWEDEN - Swedish Red Cross, Artillerigatan 6, 10440, Stockholm 14.

SWITZERLAND - Swiss Red Cross, Tauben. strasse 8, B.P. 2699, 3001 Berne.

SYRIA - Syrian Red Crescent, 13, rue Abi-Ala Almaari, Damascus.

TANZANIA - Tanzania Red Cross Society, Upanga Road, P.O.B. 1133, Dar es Salaam.

THAILAND - Thai Red Cross Society, King Chulalongkorn Memorial Hospital, Bangkok.

TOGO - Togolese Red Cross Society, Avenue des Alliés 19, P.O. Box 655, Lomé.

TRINIDAD AND TOBAGO - Trinidad and Tobago Red Cross Society, 48 Pembroke Street, P.O. Box 357, Port of Spain.

TUNISIA - Tunisian Red Crescent, 19, rue d'Angleterre, Tunis.

TURKEY - Turkish Red Crescent, Yenisehir, Ankara.

UGANDA - Uganda Red Cross, 57 Roseberry Street, P.O. Box 494, Kampala.

UNITED ARAB REPUBLIC - Red Crescent Society of the United Arab Republic, 34, rue Ramses, Cairo.

UPPER VOLTA - Upper Volta Red Cross, P.O.B. 340, Owagadougou.

URUGUAY - Uruguayan Red Cross, Avenida 8 de Octubre, 2990, Montevideo.

U.S.A. - American National Red Cross, 17th and D Streets, N.W., Washington 6 D.C.

U.S.S.R. - Alliance of Red Cross and Red Crescent Societies, Tcheremushki, J. Tcheremushkinskii proezd 5, Moscow $W$-36.

VENEZUELA - Venezuelan Red Cross, Avenida Andrés Bello No. 4, Apart. 3185, Caracas.

VIET NAM (Democratic Republic) - Red Cross of the Democratic Republic of Viet Nam, 68, rue Bà-Trièz, Hanoi.

VIET NAM (Republic) - Red Cross of the Republic of Viet Nam, 201, duong HongThâp-Tu, No. 201, Saigon.

YUGOSLAVIA - Yugoslav Red Cross, Simina ulica broj 19, Belgrade.

ZAMBIA - Zambia Red Cross, P.O. Box R. W. 1, Ridgeway, Lusaka. 